\title{
Disease-modifying Therapies for the Prevention of Type 1 Diabetes
}

\author{
An Expert Interview with Carla Greenbaum \\ Diabetes Program, Benaroya Research Institute, Seattle, Washington, USA
}

DOl: https://doi.org/10.17925/USE.2019.15.1.15

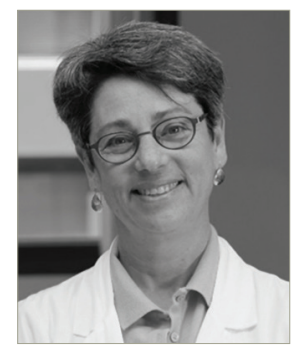

\section{Carla Greenbaum}

Carla Greenbaum received her undergraduate and medical degrees at Brown University, Rhode Island, then pursued her endocrinology fellowship at University of Washington, where she continues as clinical faculty. She joined the Benaroya Research Institute (BRI) in 2000, where she is a Member and serves as Director of the Diabetes Research Program and the BRI Clinical Research Center. Dr Greenbaum serves on the Board of Directors for BRI and is a member of the BRI Institutional Review Board, which provides oversight for clinical studies.

\section{Keywords}

Disease-modifying therapies, type 1 diabetes, TrialNet, autoimmune disease

Disclosure: Carla Greenbaum has no conflicts of interest to declare in relation to this article.

Review Process: This is an expert interview and as such, has not undergone the journal's standard peer-review process.

Acknowledgement: Medical writing assistance was provided by Katrina Mountfort of Touch Medical Media.

Compliance with Ethics: This article is an opinion piece and does not report on new clinical data, or any studies with human or animal subjects performed by any of the authors.

Authorship: The named author meets the Internationa Committee of Medical Journal Editors (ICMJE) criteria for authorship of this manuscript, takes responsibility for the integrity of the work as a whole, and has given final approval for the version to be published.

Received: February 18, 2019

Accepted: April 5, 2019

Citation: US Endocrinology, 2019;15(1):15-6

Corresponding Author: Carla Greenbaum MD, Benaroya Research Institute, Seattle, Washington, USA. E: diabetes@benaroyaresearch.org; www.trialnet.org

Facebook:www.facebook.com/DiabetesTrialNet/

Twitter: https://twitter.com/T1D_TrialNet

Support: No funding was received in

the publication of this article.
$\mathrm{T}$ ype 1 diabetes (T1D) is an autoimmune disease characterized by the dysfunction and/or destruction of the insulin-producing beta cells found in the pancreatic islets of Langerhans. ${ }^{1} \mathrm{New}$ insights into the course of T1D have led to the realization that the disease progresses through distinct stages prior to the onset of symptoms, and the development of a new staging system for diabetes, which classifies a preclinical stage of the disease. ${ }^{2}$ We have also learned that autoimmunity occurs early in life, almost always leads to symptomatic disease, and that some immune therapies can alter the disease course. ${ }^{3}$ In an expert interview, Carla Greenbaum discusses the use of diseasemodifying therapies at an early pre-clinical stage of T1D.

\section{Q: How are new models for disease progression helping us understand the need for disease-modifying therapy in type 1 diabetes?}

One of the exciting findings after more than 35 years of research, studying people who will potentially develop diabetes, is that we now know that when we test people for antibodies and find people who have two or more of these antibodies, almost all of them will eventually develop T1D.4,5 This means that we can think of having two or more antibodies as being the start of the disease. For example, when we treat people with high blood pressure, we are treating them for the early signs of a disease that will lead to heart attack or stroke. In the same way, if we can treat people for T1D at this early stage, we can slow disease progression. We want to change the thinking to treating early disease rather than preventing disease. ${ }^{5}$ Unfortunately, we don't have a name for this early disease stage but it is an autoimmune disorder that will almost inevitably lead to T1D.

\section{Q: What is the optimum time for intervention with disease-modifying therapies?}

Most of the studies that have been done with disease-modifying therapies have enrolled people soon after their clinical diagnosis of disease. ${ }^{3}$ The aims of those trials have been to assess whether we can save more of the insulin-producing beta cells if we treat with disease-modifying therapies. We now have five therapies (teplizumab, rituximab, abatacept, alefacept and antithymocyte globulin, see question five) that proved that, for a while, we can save a patient's remaining beta cells. If more of the beta cells are functioning, a patient will do better with their diabetes and there will be fewer concerns with long-term complications. The majority of the studies have been done in patients that already have clinical disease. The challenge is that preserving a patient's beta cells does not make them feel any different, and so it is difficult for people to understand how intervening at that point is important. 
By contrast, if we intervene early, when people have multiple antibodies but do not yet require insulin or have any symptoms of diabetes, we delay the development of clinical disease. This means not having to monitor blood sugar, not having to take insulin, and not having to be concerned about fluctuating blood sugar levels. One can consider therefore that the optimum time to take disease-modifying therapies is prior to diagnosis.

\section{Q: How important is screening relatives and disease modifying in people with type 1 diabetes?}

Once there is someone in your family with T1D, the risk of developing T1D among family members is 15 times greater than if you have no family members with disease. ${ }^{5}$ We know that genetics play an important role in the disease and by screening relatives we can identify people who will have multiple antibodies and will eventually progress to clinical disease if we don't intervene. When we screen people, the vast majority of relatives will not have these antibodies, and this screening can be reassuring However, among the people that do have multiple antibodies, whether or not they choose to take part in a clinical trial, one of the important benefits is that we can monitor these patients over time. By noting changes over time in glucose, we can detect the progression of T1D long before clinical symptoms occur. Often, by the time people are diagnosed they have very high blood glucose and a potentially fatal condition called diabetic ketoacidosis which can put them in hospital for several days. Participation in a screening program and regular monitoring will enable us to see the disease progressing long before these severe symptoms, and thus the onset of disease, when it does occur, is much milder and easier to control. To screen a family member, visit: trialnet.org

\section{Q: How does type 1 diabetes diagnosed in children differ from that diagnosed in adults?}

Another benefit of all the research is that we understand more about the natural history of the disease. Most people think of T1D as a disease that begins in children but we now estimate that about half the people with T1D have disease onset as adults. Nonetheless, there are differences. ${ }^{5,6}$ At each disease stage, children progress faster, i.e., a child with multiple antibodies will progress to clinical disease at a faster rate than an adult, even if they still have the same antibodies. The same is true after diagnosis; children usually start with less of their natural insulin production left compared with adults and their rate of beta-cell loss is more rapid. At present we don't understand why that is the case. It is important to know that both children and adults can get T1D, but characteristics are often different between the two.

\section{Q: What have been the most promising disease-modifying therapies for type 1 diabetes to date?}

It is very exciting to realize that we now have data from five clinical trials that have shown that these therapies can alter the disease course of T1D. These include an anti-T cell therapy called teplizumab, ${ }^{7}$ and anti-B cell therapy called rituximab; a therapy that interferes with the relationship between the two, post-stimulation blockade, called abatacept; 9 a drug that interferes with a specific type of immune cell called memory cells, which is called alefacept; ${ }^{10}$ and the most recent study, which also has a positive result, was with antithymocyte globulin. ${ }^{11}$ Each of these therapies, when given to newly diagnosed patients, were shown to preserve insulin production, and in some cases, showed that insulin preservation can last for a long time. These therapies are very important and some are already being tested in early-stage disease. Type 1 Diabetes TrialNet is a National Institutes of Health-funded and JDRF-supported international clinical trials network that aims to bring disease-modifying therapies to individuals with T1D. ${ }^{5,12}$ In June 2019 TrialNet aims to announce results of the teplizumab trial for prevention of diabetes in relatives at-risk (NCT01030861). For further information, including videos from participants, frequently asked questions, and more, visit: trialnet.org

One of the most important messages is to recognize that these are all immune therapies because T1D is an autoimmune disease. The term diabetes only refers to the metabolic consequences but T1D is more similar to diseases like multiple sclerosis, Crohn's disease, and rheumatoid arthritis. At present, T1D is one of the only autoimmune diseases that is treated solely with treatment for the symptoms, so this is an opportunity to bring diabetes to the status of other autoimmune diseases and use disease modifying therapies. $]$
1. Bach JF. Insulin-dependent diabetes mellitus as an autoimmune disease. Endocr Rev. 1994;15:516-42.

2. Insel RA, Dunne $J \mathrm{~L}$, Atkinson MA, et al. Staging presymptomatic type 1 diabetes: a scientific statement of JDRF, the Endocrine Society, and the American Diabetes Association. Diabetes Care. 2015;38:1964-74.

3. Lord S, Greenbaum CJ. Disease modifying therapies in type 1 diabetes: Where have we been, and where are we going? Pharmacol Res. 2015;98:3-8.

4. Bonifacio E. Predicting type 1 diabetes using biomarkers Diabetes Care. 2015;38:989-96.

5. Bingley PJ, Wherrett DK, Shultz A, et al. Type 1 diabetes TrialNet: A multifaceted approach to bringing disease-modifying therapy to clinical use in type 1 diabetes. Diabetes Care. 2018;41:653-61.

6. Wherrett DK, Chiang JL, Delamater AM, et al. Type 1 diabetes TrialNet study group. Diabetes Care. 2015 Oct;:38:1975-85.

7. Long SA, Thorpe J, DeBerg HA, et al. Partial exhaustion of CD8 T cells and clinical response to teplizumab in new-onset type 1 diabetes. Sci Immunol. 2016;1: pii:eaai7793.

8. Pescovitz MD1, Greenbaum CJ, Krause-Steinrauf $\mathrm{H}$, et al. Rituximab, B-lymphocyte depletion, and preservation of beta-cell function. N Eng/ J Med. 2009;361:2143-52.

9. Orban T1, Bundy B, Becker DJ, et al. Co-stimulation modulation with abatacept in patients with recent-onset type 1 diabetes: a randomised, double-blind, placebo-controlled trial. Lancet.
2011;378:412-9

10. Rigby MR, Harris KM, Pinckney A, et al. Alefacept provides sustained clinical and immunological effects in new-onset type 1 diabetes patients. J Clin Invest. 2015;125:3285-96.

11. Haller MJ, Schatz DA, Skyler JS, et al. Type 1 Diabetes TrialNet ATGGSG. Low-dose anti-thymocyte globulin (ATG) preserves betacell function and improves HDA1c in new-onset type 1 diabetes. Diabetes Care. 2018;41:1917-25.

12. Battaglia M, Anderson MS, Buckner JH, et al. Understanding and preventing type 1 diabetes through the unique working model of TrialNet. Diabetologia. 2017;60:2139-47. 